\title{
Staphylococcus aureus biofilms decrease osteoblast viability, inhibits osteogenic differentiation, and increases bone resorption in vitro
}

Carlos J Sanchez Jr ${ }^{*}{ }^{\dagger}$, Catherine L Ward ${ }^{\dagger}$, Desiree R Romano, Brady J Hurtgen, Sharanda K Hardy, Ronald L Woodbury, Alex V Trevino, Christopher R Rathbone and Joseph C Wenke

\begin{abstract}
Background: Osteomyelitis is a severe and often debilitating disease characterized by inflammatory destruction of bone. Despite treatment, chronic infection often develops which is associated with increased rates of treatment failure, delayed osseous-union, and extremity amputation. Within affected bone, bacteria exist as biofilms, however the impact of biofilms on osteoblasts during disease are unknown. Herein, we evaluated the effect of $S$. aureus biofilms on osteoblast viability, osteogenic potential, and the expression of the pro-osteoclast factor, receptor activator of NF-kB ligand (RANK-L).
\end{abstract}

Methods: Osteoblasts were exposed to biofilm conditioned media (BCM) from clinical wound isolates of Staphylococcus aureus under normal growth and osteogenic conditions to assess cellular viability and osteoblast differentiation, respectively. Cell viability was evaluated using a live/dead assay and by quantifying total cellular DNA at days $0,1,3,5$, and 7 . Apoptosis following treatment with BCM was measured by flow-cytometry using the annexin V-FITC/PI apoptosis kit. Osteogenic differentiation was assessed by measuring alkaline phosphatase activity and intracellular accumulation of calcium and osteocalcin for up to 21 days following exposure to BCM. Expression of genes involved in osteogenic differentiation and osteoclast regulation, were also evaluated by quantitative real-time PCR.

Results: BCM from clinical strains of S. aureus reduced osteoblast viability which was accompanied by an increase in apoptosis. Osteogenic differentiation was significantly inhibited following treatment with BCM as indicated by decreased alkaline phosphatase activity, decreased intracellular accumulation of calcium and inorganic phosphate, as well as reduced expression of transcription factors and genes involved in bone mineralization in viable cells. Importantly, exposure of osteoblasts to BCM resulted in up-regulated expression of RANK-L and increase in the RANK-L/ OPG ratio compared to the untreated controls.

Conclusions: Together these studies suggest that soluble factors produced by S. aureus biofilms may contribute to bone loss during chronic osteomyelitis simultaneously by: (1) reducing osteoblast viability and osteogenic potential thereby limiting new bone growth and (2) promoting bone resorption through increased expression of RANK-L by osteoblasts. To our knowledge these are the first studies to demonstrate the impact of staphylococcal biofilms on osteoblast function, and provide an enhanced understanding of the pathogenic role of staphylococcal biofilms during osteomyelitis.

Keywords: Biofilm, Osteoblast, Osteogenic differentiation, Staphylococcus aureus, Receptor activator of NF-kB ligand, Osteoprotegrin

\footnotetext{
* Correspondence: carlos.j.sanchez3@us.army.mil

${ }^{\dagger}$ Equal contributors

Department of Extremity Trauma and Regenerative Medicine, United States Army Institute of Surgical Research, Ft. Sam Houston, San Antonio, TX, USA
} 


\section{Background}

Osteomyelitis is a debilitating disease, characterized by the inflammatory destruction of bone and surrounding tissues. Disease is most commonly preceded by hematogenous spread of microorganisms to the bone from either a contiguous infection or directly following trauma. Staphylococcus aureus is the microorganism most commonly associated with hematogenous and post-traumatic osteomyelitis, accounting for more than half of all cases [1]. Despite treatment and surgical intervention up to $30 \%$ of osteomyelitis cases progress into a chronic infection [2]. Chronic osteomyelitis is associated with high rates of antimicrobial treatment failure, increased rates of non-osseous union and extremity amputation $[1,3]$. Over the past decade clinicians have adopted the 'biofilm theory' to explain the chronicity of bone infections, recalcitrance to conventional antimicrobial treatment, and incidence of infectious relapse $[4,5]$.

Biofilms are surface attached communities consisting of mono- or polymicrobial species that are surrounded by a self produced extracellular polymeric matrix [6,7]. In general, biofilms represent a protected mode of growth enabling the organisms to persist within immunocompetent hosts, and are implicated as a significant pathogenic event in the development of a number of chronic human infections, including osteomyelitis [8,9]. In support of this, studies have demonstrated the presence of staphylococcal biofilms within infected bone of patients with chronic osteomyelitis [9-11], that clinical osteomyelitis isolates of S. aureus are capable of forming biofilms in vitro [12-14], furthermore that staphylococcal biofilms are a significant factor contributing to non-union $[15,16]$. These studies indicate that staphylococcal biofilms play a critical, yet not fully understood role in the development of chronic osteomyelitis and associated infectious complications.

Osteoblasts function as the major director of net bone formation or resorption during the normal physiological turnover of bone and following infection. Osteoblasts facilitate bone formation directly, through the deposition and calcification of bone matrix promoting new bone growth, and promote bone resorption indirectly by regulating the activity of osteoclasts by the production of two cytokines, the receptor activator of NF-kB ligand (RANK-L) and osteoprotegrin (OPG), which promote and block osteoclast activity respectively [17-19]. During infection, shifts in the ratio of these two cytokines can contribute to overall bone loss, which is typical of chronic orthopaedic infections. This is largely due to the combined effects of microorganisms to elicit a local host inflammatory response and impact osteoblast function [20,21]. The current understandings of the molecular mechanisms that contribute to bone loss during osteomyelitis are based on evaluating interactions between planktonic bacteria and osteoblasts in vitro [20,22-24]. While these studies have provided insight into the bacterial mechanisms contributing to bone loss, they fail to address the role of the biofilm phenotype during disease, which is likely to be more representative of the bacterial mode of growth in vivo $[4,8,9]$. Because osteoblasts play a critical role for bone homeostasis and healing, a logical speculation is that biofilms interacting with these tissues during disease may exert their negative effects on this cell type.

In this study, we evaluated the effect of $S$. aureus biofilms on viability, osteogenic potential, and the production of RANK-L by human osteoblasts in response to biofilms. Herein, we characterize the extracellular proteome of released factors produced by $S$. aureus biofilms and demonstrate that biofilm-derived factors negatively impact cell viability and osteoblast differentiation. Furthermore, exposure of osteoblasts to biofilm-conditioned media also resulted in increased expression of RANK-L and the RANK-L/OPG ratio, indicating that biofilms simultaneously promote bone resorption. These studies are the first to show the effect of biofilms on osteoblast function and to demonstrate mechanisms through which biofilms may contribute to bone loss during chronic osteomyelitis.

\section{Methods}

\section{Bacterial strains and growth conditions}

S. aureus SAMMC-700 is a methicillin resistant clinical wound isolate of the USA300 clonal group recovered from a patient as a part of treatment and not related to research from the San Antonio Military Medical Center (SAMMC, Ft. Sam Houston, TX). UAMS-1 (ATCC strain 49230) is a methicillin-susceptible $S$. aureus strain of the USA200 clonal group, and a well characterized osteomyelitis isolate $[25,26]$. Both clinical strains were included in this study to thoroughly examine the impact of staphylococcal biofilms on osteoblast function. Bacteria were cultured in Tryptic Soy Broth (TSB) or agar plates overnight at $37^{\circ} \mathrm{C}$. For planktonic growth, individual colonies from overnight plate cultures of bacteria were used to inoculate TSB broth and were grown with agitation at $37^{\circ} \mathrm{C}$.

\section{Biofilm formation and preparation of biofilm-conditioned media (BCM)}

Biofilm formation and generation of conditioned media were performed as previously described $[27,28]$. Briefly, $500 \mu \mathrm{L}$ of a 1:100 dilution of overnight bacterial culture $\left(\sim 10^{7} \mathrm{CFU} / \mathrm{mL}\right)$ was added to transwell culture inserts (0.4 $\mu \mathrm{m}$ pore size, Corning Inc, Corning, NY) and grown under static conditions in TSB broth for $48 \mathrm{hr}$ at $37^{\circ} \mathrm{C}$. Inserts were washed for $1 \mathrm{hr}$ in $1 \times$ phosphate buffered saline (PBS), placed into Dulbecco's Modified Eagle's Medium (DMEM) (Invitrogen, Carlsbad, CA) and maintained in 
DMEM at $37^{\circ} \mathrm{C}$ for an additional 4 days. BCM was collected daily and replaced with fresh media. Pooled BCMs were filter-sterilized using a $0.2 \mu \mathrm{m}$ syringe filter (EMD Millipore, Billerica, MA), pH adjusted to 7.4, and stored at $-80^{\circ} \mathrm{C}$ until use. Sterility of collected BCM was assessed by spot plating and overnight culture of the plates. Total protein from pooled BCM was quantified using the bicinchonic acid (BCA) assay (Pierce, Rockford, IL) following the manufacturers protocol. Because S. aureus SAMMC-700 is an uncharacterized clinical wound isolate, further characterization by mass spectroscopy was pursued for this BCM. The BCM of $S$. aureus UAMS-1 was included for cellular experiments for a more extensive study.

\section{Scanning electron microscopy (SEM)}

For SEM analysis tissue culture inserts were excised, fixed with $2 \%(\mathrm{w} / \mathrm{v})$ glutaraldehyde, $2 \%(\mathrm{w} / \mathrm{v})$ paraformaldehyde (PFA), $0.15 \mathrm{M}$ sodium cacodylate, $0.15 \%(\mathrm{w} / \mathrm{v})$ alcian blue for $3 \mathrm{hr}$, rinsed $3 \times$ with $0.15 \mathrm{M}$ sodium cacodylate buffer, and incubated in $1 \%(\mathrm{v} / \mathrm{v})$ osmium tetroxide in sodium cacodylate for $1 \mathrm{hr}$. Samples were dehydrated with a stepwise gradient of ethanol and then treated with hexamethyldisilizane prior to drying in a desiccator overnight. Samples were sputter coated with gold palladium and viewed with a JEOL-6610 scanning electron microscope (JEOL USA, Inc., Peabody, MA).

\section{Protein identification by mass spectrometry}

Cell lysates were loaded onto a pre-cast 12\% SDS-PAGE gel (Bio-Rad, Hercules, CA) in Laemmli sample buffer and run $2 \mathrm{~cm}$ into the gel as measured from the bottom of the well. The gel was fixed and stained for $60 \mathrm{~min}$ using colloidal coomassie and de-stained overnight in water. Lanes were evenly cut into 6 slices starting at the top of the resolving gel and ending at the dye front $(\sim 1$ $\mathrm{cm})$. Each slice was further diced into $1 \mathrm{~mm} \times 1 \mathrm{~mm}$ cubes. Slices were then digested in situ with trypsin according to standard protocols based on the initial work of Mann and co-workers [29]. Briefly, protein bands were excised from the gel and destained twice in $50 \%$ acetonitrile $(\mathrm{ACN}) / 40 \mathrm{mM}$ ammonium bicarbonate, $\mathrm{pH}$ 7.4, prior to digestion. Gel plugs were then dehydrated in $100 \% \mathrm{ACN}$ and rehydrated with 5 to $10 \mu \mathrm{l}$ of $10 \mathrm{ng} / \mu \mathrm{l}$ trypsin (Promega; modified) in $40 \mathrm{mM}$ ammonium bicarbonate $/ 20 \% \mathrm{ACN}$ and incubated overnight at $30^{\circ} \mathrm{C}$. The resulting peptides were extracted in 4 volumes of $0.1 \% \mathrm{TFA} / 50 \% \mathrm{ACN}$ for 1 to $2 \mathrm{hr}$ at RT, decanted from the gel slice, dried down in an autosampler tube in the speed vacuum w/o heat, and resuspended in $0.5 \%$ TFA. Peptides were analyzed by capillary-HPLC-electrospray tandem mass spectrometry (HPLC-ESI-MS/MS) on a Thermo Fisher LTQ ion trap mass spectrometer coupled to an Eksigent NanoLC micro HPLC by means of a PicoView (New Objective) nanospray interface. Capillary on-line HPLC separation of tryptic peptides was conducted using the following conditions: column, New Objective PicoFrit, $75 \mu \mathrm{m}$ id, packed to $11 \mathrm{~cm}$ with $\mathrm{C} 18$ adsorbent, (Vydac 218MSB5); mobile phase A, 0.5\% acetic acid/ $0.005 \%$ TFA in water; mobile phase $\mathrm{B}, 90 \% \mathrm{ACN} /$ $0.5 \%$ acetic acid $/ 0.005 \%$ TFA in water; gradient, $2 \% \mathrm{~B}$ to $42 \% \mathrm{~B}$ in $30 \mathrm{~min}$; flow rate, $0.4 \mu \mathrm{l} / \mathrm{min}$. A data-dependent acquisition protocol was employed consisting of one survey scan followed by 7 collision-induced dissociation spectra. The un-interpreted CID spectra were searched against the NCBInr database using Mascot (Matrix Science; 10 processor in-house license). Methionine oxidation was the only variable modification considered. Maximum missed cleavages for trypsin was set at 1 , peptide charge at $2+$ and $3+$, peptide tolerance at $+/-1.5 \mathrm{Da}$, and MS/MS tolerance at + /- 0.8 Da. Mascot data was then run in Scaffold 3.1 (www.proteomesoftware.com) and cross correlation of the Mascot results was carried out by $\mathrm{X}$ ! tandem against the NCBInr subset database. Proteins with an expectation score of $10^{-3}$ or lower were considered positive identities. Proteins were identified with 3-15 matched peptides and a minimum of $95 \%$ sequence coverage.

\section{Culture of human osteoblasts and osteogenic differentiation}

Human osteoblasts (PromoCell, Heidelberg, Germany) were maintained in DMEM supplemented with $10 \%$ fetal bovine serum (FBS), penicillin $10 \mathrm{U} \mathrm{mL}^{-1}$ and streptomycin $10 \mu \mathrm{g} \mathrm{mL}{ }^{-1}$ at $37^{\circ} \mathrm{C}$ in $5 \% \mathrm{CO}_{2}$. For osteogenic differentiation of osteoblasts cells were seeded into 24-well plates or $10 \mathrm{~cm}$ culture dishes and when cells reached $80 \%$ confluence they were cultured in media supplemented with ascorbic acid $(50 \mu \mathrm{M}), \beta$-glycerolphosphate $(20 \mathrm{mM})$ and dexamethasone $(1.5 \mu \mathrm{M})$ (Sigma, St. Louis, $\mathrm{MO})$ for up to 21 days in the presence or absence of BCM.

\section{Cell viability and proliferation}

Cell viability and proliferation of osteoblasts was assessed using a LIVE/DEAD ${ }^{\odot}$ assay kit and by quantifying total cellular DNA using the CyQUANT ${ }^{\circledR}$ Cell Proliferation Assay (Molecular Probes, Grand Island, NY), respectively, following exposure to BCM up to 21 days in 48-well plates as recommended by the manufacturer.

\section{Measurement of apoptosis in osteoblasts}

Apoptosis in osteoblasts was evaluated after $24 \mathrm{hr}$ treatment with BCM using the Annexin V-FITC apoptosis detection kit (BD, Franklin Lakes, NJ) by fluorescenceactivated cell sorting (FACS) using a FACSCalibur flow cytometer (BD, Franklin Lakes, NJ) as previously described [30]. Data was analyzed using FlowJo software (Tree Star, Inc., Ashland, OR). As a positive control for apoptosis, $4 \mu \mathrm{M}$ staurosporine (Sigma, St. Louis, MO) was used [31]. 


\section{Alkaline phosphatase (ALP) measurement}

Quantification of ALP activity in osteoblasts was carried out using the SensoLyte ${ }^{\circledR}$ pNPP Alkaline Phosphatase Assay (AnaSpec, Fremont, CA) according the manufacturer's protocol. Briefly, cells were lysed using Triton $\mathrm{X}-100$, and alkaline phosphatase activity was detected within supernatants by measuring absorbance of the dephosphorylated chromogenic substrate, p-Nitrophenyl phosphate, at $405 \mathrm{~nm}$. Concentrations of ALP within the supernatants were determined using a standard curve generated with calf intestinal ALP.

\section{Osteocalcin staining}

Osteoblasts were grown and differentiated for 14 and 21 days in 24-well plates in the presence or absence BCM as above. Cells were then fixed with 4\% PFA, permeabilized and blocked with $1 \%$ BSA $+0.1 \%$ Triton X-100 in $1 \times$ PBS for $30 \mathrm{~min}$. Mouse anti-human antibody for osteocalcin (R\&D systems, Minneapolis, MN) was diluted 1:200 and added to cells for $1 \mathrm{hr}$. Phycoerythrin (PE) labeled secondary goat anti-mouse antibody (Abcam, Cambridge, MA) was added at a final concentration of 1:10,000 and cells were visualized with an Olympus IX71 inverted fluorescence microscope (Olympus Inc, Center Valley, PA).

\section{Alizarin red S staining}

Intracellular calcium deposition in osteoblasts following treatment with BCM under differentiating conditions was assessed by Alizarin Red staining. Briefly, osteoblasts were seeded into 24-well plates and grown under osteogenic conditions as described above in the presence or absence of BCM. At 7, 14, and 21 days cells were fixed with $4 \%$ PFA, washed with sterile $1 \times$ PBS, stained with $2 \%(\mathrm{w} / \mathrm{v})$ Alizarin Red S (Sigma, St. Louis, MI) and visualized by light microscopy.

\section{RNA extraction and real-time PCR}

RNA was extracted from cells treated with BCM in $10 \mathrm{~cm}$ cell culture dishes, processed with the QIAshredder, and purified using the RNAeasy Mini Kit (Qiagen, Valencia, CA) as per manufacturer instructions. First strand synthesis was achieved with SuperScript III first-strand synthesis supermix with oligo-dT primers (Invitrogen, Carlsbad, CA) for each RNA sample following recommended protocols using a PTC-100 Thermal Cycler (GMI Inc, Ramsey, $\mathrm{MN})$. For genes of interest (Table 1), quantitative realtime polymerase chain reaction (qRT-PCR) was performed using a Bio-Rad C1000 system and analyzed using iQ5 software (BioRad, Hercules, CA). The primers sets used in this study were based on optimized and validated primers from PrimerBank ${ }^{\odot}$ (Table 1). Amplification reactions were performed using qPCR iQSYBR Green Super Mix (BioRad, Hercules, CA) with the following conditions: 10 min at $95^{\circ} \mathrm{C}$, followed by 40 cycles at $95^{\circ} \mathrm{C}$ for $10 \mathrm{~s}, 60^{\circ} \mathrm{C}$ for $30 \mathrm{~s}, 72^{\circ} \mathrm{C}$ for $30 \mathrm{~s}$. Three independent biological experiments with three technical replicates were performed for each reaction. Transcript levels were normalized to the internal control, Glyceraldehyde-3-phosphate dehydrogenase (GAPDH), mRNA and changes in relative expression were calculated using $2^{-\Delta \mathrm{Ct}}$ method [32].

Table 1 Primers used in this study ${ }^{a}$

\begin{tabular}{lcl}
\hline Gene & Accession number & Primer sequence \\
\hline Activating transcription factor 4 & ATF & Sense CCCTTCACCTTCTTACAACCTC \\
Runt-related transcription factor 2 & (NM_182810) & Antisense TGCCCAGCTCTAAACTAAAGGA \\
Alkaline phosphatase & RUNX2 & Sense TGGTTACTGTCATGGCGGGTA \\
& $($ NM_001015051) & Antisense TCTCAGATCGTTGAACCTTGCTA \\
Osteocalcin & ALP & Sense AACATCAGGGACATTGACGTG \\
& (NM_001127501) & Antisense GTATCTCGGTTGAAGCTCTTCC \\
Osteonectin & BGLAP & Sense CACTCCTCGCCCTATTGGC \\
Receptor Activator of NF-KB Ligand & (NM_000711) & Antisense GCCTGGGTCTCTTCACTACCT \\
Osteoprotegerin & SPARC & Sense CCCATTGGCGAGTTGAGAAG \\
& (NM_003118) & Antisense AGGAAGAGTCGAAGGTCTTGTT \\
Glyceraldehyde-3-phosphate & RANK-L & Sense GTCTGCAGCGTCGCCCTGT \\
& $($ NM_003701) & Antisense ACCATGAGCCATCCACCATCGC \\
\hline
\end{tabular}

${ }^{a}$ Primers used in this study were obtained from Primer Bank "PCR Primers for gene Expression Detection and Quantification". http://pga.mgh.harvard.edu/ primerbank/ (The Center for Computational and Integrative Biology). All selections were made relative to comparable annealing temperatures, as well as products close to $100 \mathrm{bp}$ in length to maintain consistent PCR conditions. 


\section{Statistical analysis}

Statistical analyses were performed using One-way ANOVA with appropriate post-hoc tests for comparisons between groups using sigmaplot version 12.0. $P$-values of $<0.05$ were considered to be statistically significant.

\section{Results}

Characterization of biofilm-derived factors produced by clinical isolates of $S$. aureus

After 48 hours of growth under static conditions, both $S$. aureus strain UAMS-1 and SAMMC-700 formed mature biofilms on the transwell surface. As shown in the representative SEM images, mature biofilms of both strains were characterized by large heterogeneous structures composed of accumulations of aggregated bacteria, with few visible planktonic bacteria, confirming that the majority of cells using this model were in a biofilm state (Figure 1A). BCM on average contained $32 \pm 5$ and $25 \pm$ $6 \mu \mathrm{g} / \mathrm{mL}$ of extracellular protein for UAMS-1 and
SAMMC-700 respectively (Figure 1B). Given the similarity in protein concentrations between the BCM produced by both strains; moreover that UAMS-1 is a well characterized isolate, we choose to characterize the $\mathrm{BCM}$ of the wound isolate SAMMC-700 by mass spectrometry to identify those soluble factors present. Proteomic analysis of the biofilm conditioned media (BCM) from $S$. aureus SAMMC-700, demonstrated the presence of a heterogeneous mixture of proteins encompassing all aspects of bacterial physiology, including transcription, translation, energy metabolism, pathogenesis, and proteins of unknown function (Figure 1C; Additional file 1). The predominate class of extracellular proteins present within the BCM were primarily those involved in energy metabolism (42\%), including carbohydrate, lipid, and nucleotide metabolism, as well as those involved in protein synthesis and processing (19\%). Interestingly, although virulence factors were detected within the BCM of $S$. aureus SAMMC-700, this group represented a
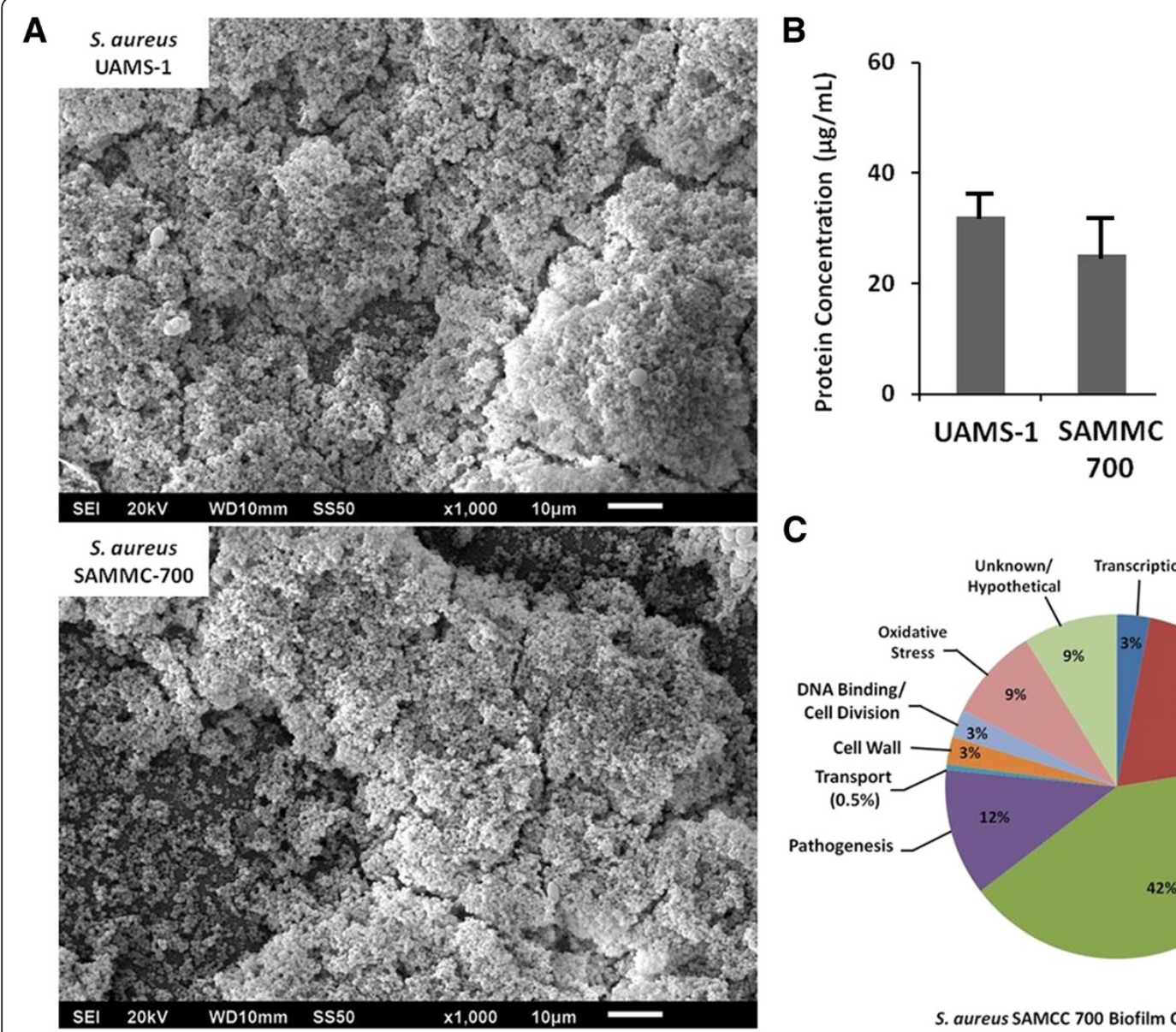

C

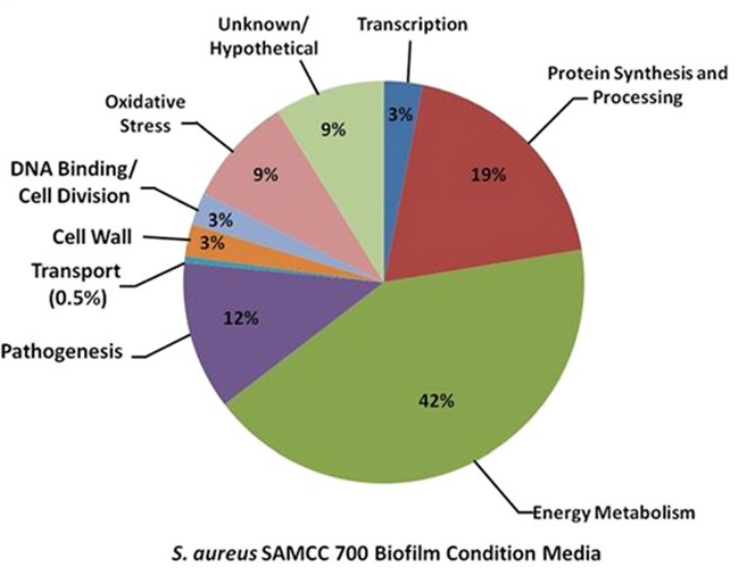

Figure 1 Soluble factors produced by Staphylococcus aureus biofilms. A) Representative SEM images of S. aureus strain UAMS1 (top panel) and SAMMC -700 (bottom panel) after $48 \mathrm{hr}$ growth (scale bars $=10 \mu \mathrm{m})$. B) Total protein $(\mu \mathrm{g} / \mathrm{mL}$ ) within the BCMs of S. aureus clinical strains as determined by the BCA assay. C) Proteomic analysis of BCM from S. aureus strain SAMMC-700 by mass spectroscopy. Proteins identified within the $\mathrm{BCM}$ were separated into functional categories, and represented as a percentage of the total proteins identified. 
small percentage of the total extracellular proteome (12\%; Additional file 1).

\section{Biofilm-derived factors reduce osteoblast viability by activating apoptosis}

BCMs from S. aureus UAMS-1 and SAMMC-700 had a dose-dependent effect on the viability of human osteoblasts, with concentrations $\geq 50 \%$ having a significant, detrimental effect on cell viability within $24 \mathrm{hr}$. In contrast, at $25 \%$ minimal effects on osteoblast viability were observed albeit increasing over time with maximal effect at 21 days, whereas concentrations $25 \%$ had little to no effect at $24 \mathrm{hr}$ (Additional files 2, 3). Based on the viability studies and to permit the adequate time required to evaluate osteogenic differentiation, we chose to use the $50 \%$ BCM for short term ( $\leq 7$ days) studies, including viability and apoptosis, and 25\% BCM for extended experiments ( $\geq 7$ days), those related to evaluating osteogenic differentiation. Exposure of osteoblasts to the BCM from S. aureus SAMMC-700 significantly reduced cell viability and proliferation as determined by measuring the amount of viable cells and quantification of cellular DNA, respectively (Figure 2A-B). Evaluation of osteoblasts, following treatment with BCM for $24 \mathrm{hr}$ by Annexin $\mathrm{V}$ staining, demonstrated that BCM of $S$. aureus was capable of inducing apoptosis (Figure 2C-D). These results suggest that the decreases in cell number may have been due, at least in part, to an increase in apoptosis. Importantly, the effects of BCM on osteoblasts were not strain independent, as similar effects on viability and activation of apoptosis were observed when using the BCM of S. aureus strain UAMS-1 (Figure 2A-D).
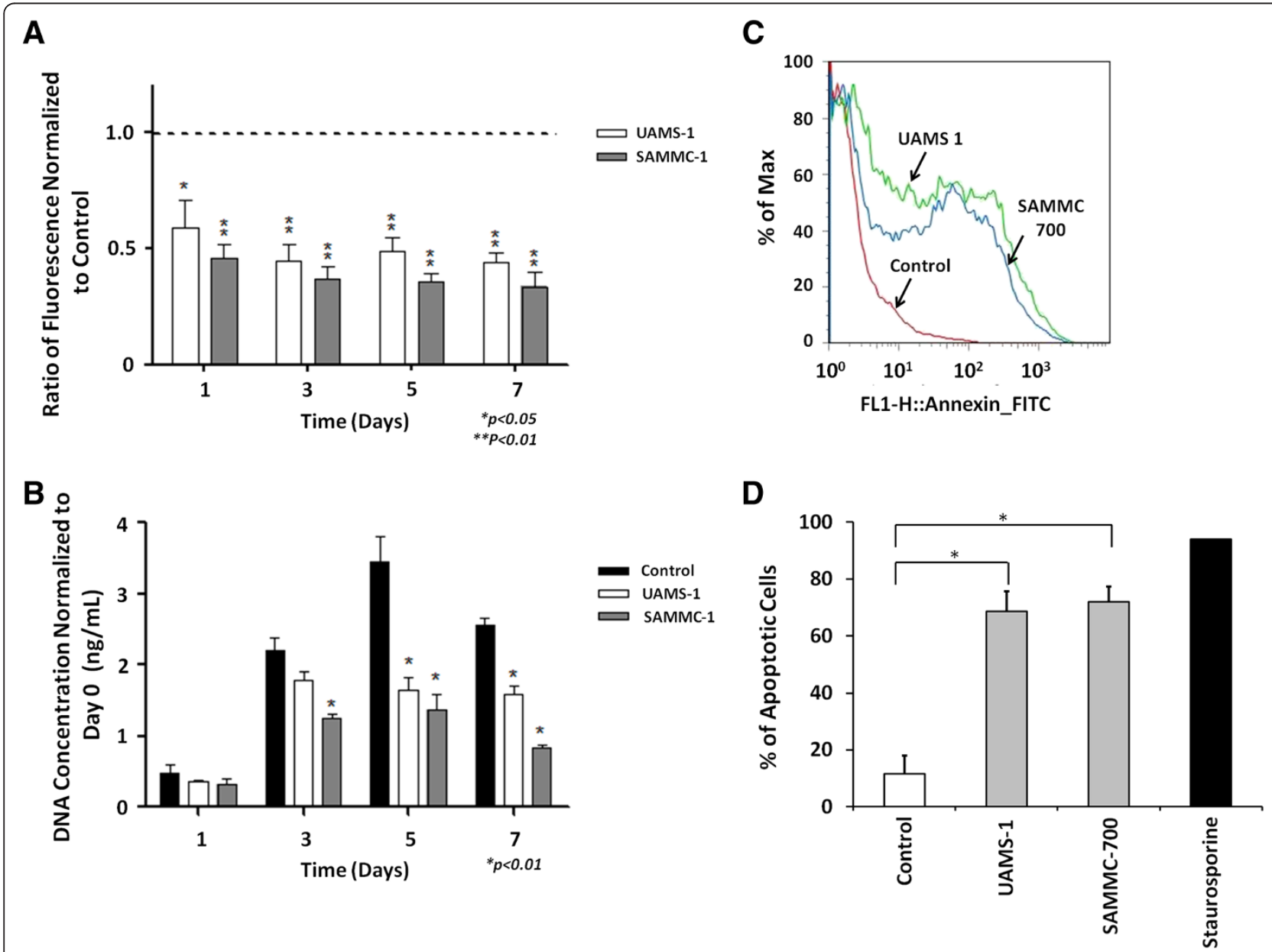

Figure 2 Biofilm factors reduce viability and activate apoptosis in human osteoblasts. A) Viability in osteoblast expressed as ratio of fluorescence (495ex/515em) normalized to non-treated control group B) Total DNA recovered from osteoblasts following treatment with BCM, normalized to day 0 controls. Bars represent experimental averages of three independent experiments \pm standard deviation. C) Representative flow-cytometry histograms measuring apoptosis in osteoblasts exposed to BCM for 24 hr by Annexin V staining and D) corresponding percentages of Annexin $V$ positive cells from two independent experiments. Statistical analysis was performed using a One-Way ANOVA with a Bonferroni test to determine statistical differences between groups; ${ }^{*} p<0.01,{ }^{* *} p<0.001$ versus controls. 


\section{Staphylococcal biofilm factors inhibit osteogenic differentiation in vitro}

To evaluate the effect of soluble factors produced by biofilms on osteogenic differentiation, a critical function of this cell type, osteoblasts were cultured under osteogenic conditions in the presence or absence of $\mathrm{BCM}$ for up to 21 days. The addition of $\mathrm{BCM}$ from the two clinical strains of $S$. aureus dramatically reduced ALP activity (Figure 3A) as well as the intercellular accumulation of calcium and osteocalcin (Figure 3B, C) in viable cells when compared to the osteogenic control group. Consistent with these results, gene expression analysis of the transcriptional regulators involved in osteogenic differentiation, including activating transcription factor 4 (Atf4), runt-related transcription factor 2 (Runx2), as well as those genes involved in matrix mineralization, alkaline phosphatase $(A l p)$, osteocalcin (Bglap) and osteonectin (Sparc) by qRT-PCR were also observed to be significantly decreased at days 7 and 14 following treatment with the $\mathrm{BCM}$ in viable cells (Table 2).

\section{Biofilm conditioned medias promote bone resorption by} up regulating RANK-L expression and increasing the RANK-L/OPG ratio

To assess the effect of staphylococcal biofilm factors on RANK-L and OPG expression in osteoblasts, we evaluated relative gene expression by qRT-PCR analysis following exposure to $\mathrm{BCM}$. At 1, 3, 7 and 14 days relative levels of RANK-L expression in osteoblasts were significantly increased in comparison to the control group following exposure to the BCM of both $S$. aureus strains (Figure 4A). Relative expression of OPG was increased at day 3 but not at any of the other days evaluated for UAMS-1(Figure 4B). No significant increases of OPG were observed in osteoblasts treated with BCM from $S$. aureus strain SAMMC-700. Importantly, compared to

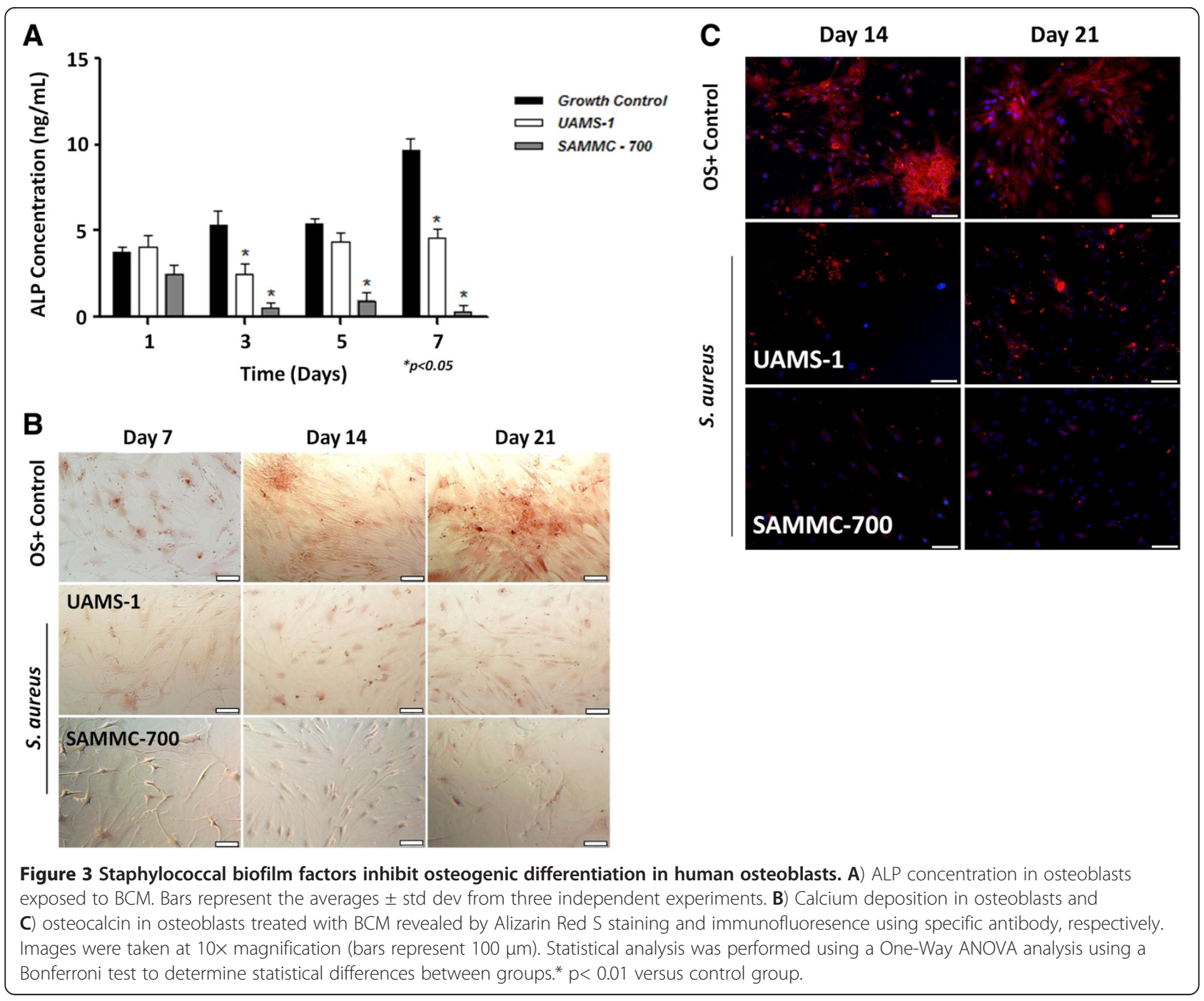


Table 2 Relative expression of genes involved in osteogenic differentiation

\begin{tabular}{|c|c|c|c|c|}
\hline & \multicolumn{2}{|c|}{ Day 7} & \multicolumn{2}{|c|}{ Day 14} \\
\hline & Relative expression & Fold difference $^{a}$ & Relative expression & Fold difference $^{\mathrm{a}}$ \\
\hline \multicolumn{5}{|l|}{ Transcription factors } \\
\hline \multicolumn{5}{|l|}{ runx2 } \\
\hline No Treatment (+ osteogenic media)* & $1.12 \pm 0.10$ & 5.333 & $2.55 \pm 0.23$ & 3.643 \\
\hline UAMS-1 & $0.12 \pm 0.18$ & 0.590 & $0.93 \pm 0.22$ & 1.329 \\
\hline SAMMC-700 & $0.08 \pm 0.25$ & 0.381 & $0.13 \pm 0.80$ & 0.186 \\
\hline \multicolumn{5}{|l|}{ atf4 } \\
\hline No Treatment (+ osteogenic media)* & $35.4 \pm 10.0$ & 2.476 & $72.1 \pm 0.30$ & 3.433 \\
\hline UAMS-1 & $16.1 \pm 0.15$ & 1.126 & $26.1 \pm 1.00$ & 1.243 \\
\hline SAMMC-700 & $13.5 \pm 0.16$ & 0.944 & $19.3 \pm 0.21$ & 0.919 \\
\hline \multicolumn{5}{|l|}{ Genes involved in mineralization } \\
\hline \multicolumn{5}{|l|}{ alp } \\
\hline No Treatment (+ osteogenic media)* & $5.73 \pm 0.14$ & 6.374 & $9.01 \pm 0.04$ & 15.615 \\
\hline UAMS-1 & $0.17 \pm 0.01$ & 0.187 & $1.12 \pm 0.04$ & 2.166 \\
\hline SAMMC-700 & $0.27 \pm 0.01$ & 0.306 & $0.21 \pm 0.10$ & 0.406 \\
\hline \multicolumn{5}{|l|}{ bglap } \\
\hline No Treatment (+ osteogenic media)* & $0.77 \pm 0.04$ & 6.814 & $6.0 \pm 0.33$ & 40.816 \\
\hline UAMS-1 & $0.001 \pm 1.00$ & 0.009 & $0.10 \pm 0.33$ & 0.680 \\
\hline SAMMC-700 & $0.001 \pm 0.51$ & 0.009 & $0.01 \pm 0.34$ & 0.068 \\
\hline \multicolumn{5}{|l|}{ sparc } \\
\hline No Treatment (+ osteogenic media)* & $174 \pm 37.8$ & 2.568 & $124 \pm 0.21$ & 1.879 \\
\hline UAMS-1 & $44.5 \pm 22.7$ & 0.656 & $48.7 \pm 0.24$ & 0.738 \\
\hline SAMMC-700 & $35.7 \pm 24.2$ & 0.526 & $84.9 \pm 0.25$ & 1.286 \\
\hline
\end{tabular}

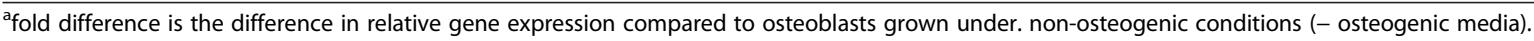

*Indicates a positive osteogenic differentiation control; osteogenic media no BCM treatment.

the control group BCM from both strains of S. aureus significantly increased the RANK-L/ OPG ratio in osteoblasts at days 1,7 and 14 (Figure $4 C$ ). BCMs of $S$. aureus strains SAMMC-700 and UAMS-1 increased the RANK-L/OPG ratio 3.7 - and 4.6 -fold at day 1 , an average of 1.5 -fold at day 3 , and averages of $>40$ fold at days 7 and 14 .

\section{Discussion}

Bone regeneration following normal physiological turnover and pathological events requires coordinated responses directed by osteoblasts to promote adequate resorption and new bone growth. The depletion of osteoblasts, inhibition of osteogenic potential, or dysregulation of bone resorption by microorganinisms during infection, such as $S$. aureus, can have detrimental effects on osseous regeneration. The current understanding of pathological mechanisms of bone loss during bacterial disease is from studies evaluating interactions between planktonic bacteria and osteoblasts [20,24,33-35]. Although these studies have provided insight into some of the pathological mechanisms contributing to bone loss they do not address the role of the biofilm during disease. This is a particularly important lapse, given that staphylococcal biofilms are considered to be the predominate mode of growth in affected bone in vivo, and have a central, yet not fully understood role, in the development of chronic osteomyelitis $[4,8,15,16]$.

Microbial biofilms represent a significant barrier to wound healing [36-38]. The ability of biofilms to delay wound healing is well characterized and has been shown, in part, to be a direct effect of soluble bacterial products released from the biofilm on host cells $[27,39,40]$. For example, supernatants from $S$. aureus biofilms have been shown to reduce cellular migration and induce apoptosis in fibroblasts and keratinocytes [27,39]. Likewise, supernatants from Pseudomonas aeruginosa and Enterococcus $s p p$. have also been shown to inhibit keratinocyte migration and other wound healing functions [41]. Osteoblasts are the primary cell type involved in facilitating new bone growth and the depletion of this cell type during chronic disease can have a tremendous impact on healing outcome. The exposure of osteoblasts to BCM of S. aureus was observed to significantly reduce viability, which was in part, due to activation of apoptosis. Previous studies have shown that when co-cultured with planktonic S. aureus, 

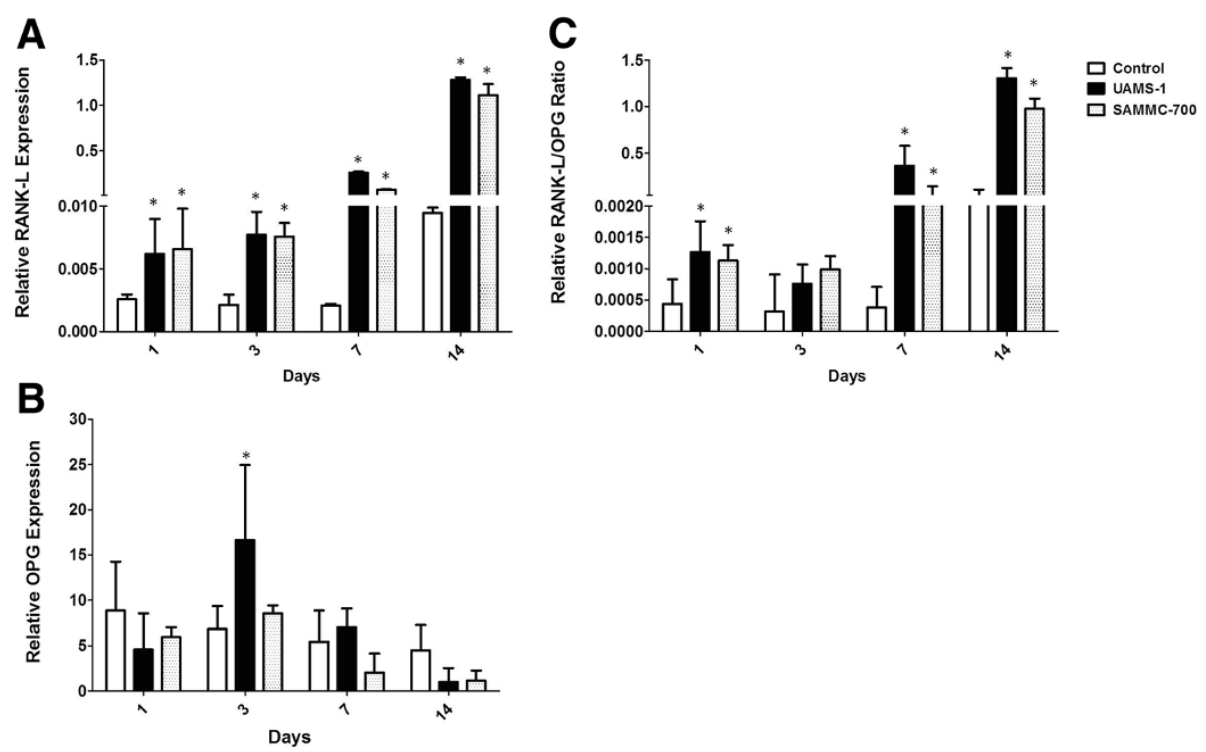

Figure 4 Biofilm-derived factors increase the expression of RANK-L and the RANK-L/OPG ratio in human osteoblasts. Relative gene expression of A) RANK-L, B) OPG, and C) the RANK-L/OPG ratio in osteoblasts exposed to BCM for 1, 3,7 and 14 days. Gene expression levels were measured by qRT-PCR, normalized to the internal control GAPDH, and presented as relative expression using the $2^{-\Delta C T}$ method. Bars represent averages \pm standard deviation from three independent experiments. Statistical analysis was performed using a One-Way ANOVA analysis using a Bonferroni test to determine statistical differences between groups at each timepoint; ${ }^{*} p<0.01$ versus control group.

osteoblasts undergo apoptosis following internalization of the bacterium, due to activation of TNF-related apoptosis inducing ligand (TRAIL) [35,42], and more recently through a family of nucleotide binding domain leucinerich repeat region (NLRs) proteins, including NLRP-3 [43]. In contrast to studies with planktonic bacteria, our results indicate that $S$. aureus biofilms activated apoptosis in osteoblasts through a paracrine effect. As TRAIL and NLRP-3 activation require active infection of the host cell, these findings indicate that a separate mechanism may be involved in activating apoptosis in osteoblasts following exposure to soluble factors produced by biofilms. Future studies are necessary to characterize the mechanisms through which apoptosis is activated in osteoblasts following exposure to BCM.

Analysis of the extracellular proteome of the BCM of S. aureus strain SAMMC-700 demonstrated the presence of a heterogeneous mixture of proteins. This included proteins primarily involved in energy metabolism, protein synthesis and to a lesser extent those involved in virulence, oxidative stress, and transcription (Additional file 1). The predominance of cytosolic proteins within the BCM is likely the result of the bacterial autolysis that occurs naturally during the maturation of the biofilm $[44,45]$. Although relationships between bacterial metabolism and virulence have been reported, soluble virulence factors, including alpha $(\alpha)$-hemolysin, gamma $(\gamma)$-hemolysin and staphopain $\mathrm{B}$, detected within the BCM may represent those factors likely contributing to cellular apoptosis. Staphylococcus aureus produces a number of different components contributing to cellular toxicity, including exoenzymes and exotoxins. Exotoxins produced by $S$. aureus include a family of four membrane-damaging hemolysins (alpha-, beta-, gamma-, and delta-hemolysin) of which $\alpha$ hemolysin ( $\mathrm{Hla}$ ) plays an important role in the pathogenesis [46]. Alpha-toxin is a pore-forming hemolytic toxin that causes membrane damage and is capable of inducing apoptosis in a wide range of mammalian cells including, keratinocytes [47], fibroblasts [48], and monocytes [49]. Likewise, the staphylococcal cysteine proteinase, staphopain B (SspB) critically impairs antibacterial functions and induces cell death in neutrophils and monocytes $[50,51]$. The impact of staphylococcal hemolysin or staphopain on osteoblast has not been previously described, and may represent an uncharacterized role for these virulence factors during osteomyelitis. Although the activation of apoptosis in osteoblasts following exposure to BCM is strongly suggestive of a role for cytolysins and/ or the staphopain this does not exclude the possibility that other metabolites and/or proteins released by the biofilms may contribute to the effects on osteoblasts. The identification of those factors that mediate these effects on osteoblasts are currently underway.

Osteoblasts facilitate new bone growth by depositing and facilitating the calcification of bone matrix. During osteoblast differentiation, several markers are expressed including alkaline phosphatase, which are important for bone matrix deposition and mineralization, as well as regulators of matrix calcification, including osteocalcin and osteonectin. Notably, the differentiation of osteoblasts is highly 
regulated by the temporal activation of various transcriptional regulators of which RUNX2 is best described [52]. RUNX2 is a transcription factor that controls skeletal development by regulating osteoblast differentiation and expression of many extracellular matrix protein genes during osteogenesis, including alkaline phosphatase $(A l p)$ and osteocalcin (Bglap) [52,53]. In the presence of BCM osteogenic differentiation was significantly impaired. Based on previous studies indicating the crucial role of the transcriptional regulators, Cbfa1/Runx2, during osteogenic differentiation our results suggest that BCMs may affect osteoblast function by decreasing the expression of Runx 2 in viable, non-apoptotic cells. Whether other pathways involved in osteogenic differentiation, including WNT signaling among others, are impacted by BCM remain to be determined.

In addition to supporting bone growth, osteoblasts also regulate bone resorption. This is accomplished through indirect control of osteoclast activity through the relative ratio of RANK-L/OPG. In the presence of BCM, RANK-L expression and the RANK-L/OPG ratios were significantly increased compared to control groups as early as day. Increased expression of RANK-L and/or changes in RANK-L/OPG ratio have been shown to be strong predictors of rapid and persistent bone loss in rheumatoid arthritis, osteoporosis, and periodontal disease [22,54]. Consequently, during disease biofilms, in addition to reduced osteogenesis, simultaneously may promote bone loss through activation of osteoclast activity as a result of increased RANK-L production by osteoblasts. The combined effects of biofilms on osteoblasts can have a significant impact on disease progression and the healing outcome in the patient.

\section{Conclusion}

Biofilm formation is a central event in the development of chronic bone infections and has been shown to be a significant contributing factor to non-osseous union in patients with osteomyelitis. The results from this study indicate that soluble biofilm factors from clinical isolates of $S$. aureus can affect bone formation and resorption through simultaneous mechanisms including: 1) reducing cellular viability through apoptosis, 2) inhibition of osteogenic differentiation, and 3) increasing RANK-L expression, promoting bone resorption. These studies are the first to demonstrate the impact of biofilms on osteoblasts; moreover to provide insights into the pathogenic mechanisms of biofilms that contribute to infectious complications during chronic bone infections.

\section{Additional files}

Additional file 1: Table S1. Soluble factors identified within BCM of S. aureus SAMMC-700 by mass spectroscopy.
Additional file 2: Dose dependent response of BMC on osteoblast viability. osteoblasts were exposed to various concentrations $(5,10,15$, $25,50,75$ and $100 \%$ ) of BMC for up to 3 days. Viability following exposure was determined by measuring fluorescence (495ex/515em) and expressed as a ratio to the non-treated control group.

Additional file 3: Effect of biofilm conditioned media (BMC) on Osteoblast Viability. Osteoblast were exposed to BMC at 25\% for up to 21 days. Viability following exposure was determined by measuring fluorescence $(495 \mathrm{ex} / 515 \mathrm{em})$ and expressed as a ratio to the non-treated control group.

Competing interests

The authors declare that they have no competing interests.

\section{Authors' contributions}

CJS participated in the study design, experimental studies and data analysis, and wrote the final draft of this manuscript. CLW participated in the study design, experimental studies, data analysis, and in reviewing manuscript DRR, BJH, SKH, RLW and AVT performed the experimental studies and acquisition of data. CRR and JCW participated in the study design, data analysis, and helped review the manuscript. All authors read and approved the final version of the manuscript.

\section{Acknowledgments}

This work was supported by intramural funding from the Combat Casualty Research Program, Medical Research and Materiel Command to JCW. CJS, CLW and BJH are supported by a Post-doctoral Research Associate Fellowship through the National Academy of Sciences.

\section{Disclaimer}

The views expressed herein are those of the authors and do not reflect the official policy or position of the Department of the Army, Department of Defense, or the US Government. The authors are employees of the US government. This work was prepared as part of their official duties and, as such, there is no copyright to be transferred.

Received: 31 January 2013 Accepted: 6 June 2013

Published: 14 June 2013

\section{References}

1. Lew DP, Waldvogel FA: Osteomyelitis. Lancet 2004, 364:369-379.

2. Conterno LO, da Silva Filho CR: Antibiotics for treating chronic osteomyelitis in adults. Cochrane Database Syst Rev 2009, 3:CD004439. doi:10.1002/14651858.CD004439.pub2.

3. Huh J, Stinner DJ, Burns TC, Hsu JR: Infectious complications and soft tissue injury contribute to late amputation after severe lower extremity trauma. J Trauma 2011, 71:S47-S51

4. Costerton JW: Biofilm theory can guide the treatment of device-related orthopaedic infections. Clin Orthop Relat Res 2005, 437:7-11.

5. Costerton JW, Stewart PS, Greenberg EP: Bacterial biofilms: a common cause of persistent infections. Science 1999, 284:1318-1322.

6. Costerton JW: Introduction to biofilm. Int J Antimicrob Agents 1999, 11:217-221. discussion 237-219.

7. Hall-Stoodley L, Costerton JW, Stoodley P: Bacterial biofilms: from the natural environment to infectious diseases. Nat Rev Microbiol 2004, 2:95-108.

8. Brady RA, Leid JG, Calhoun JH, Costerton JW, Shirtliff ME: Osteomyelitis and the role of biofilms in chronic infection. FEMS Immunol Med Microbiol 2008, 52:13-22.

9. Marrie TJ, Costerton JW: Mode of growth of bacterial pathogens in chronic polymicrobial human osteomyelitis. J Clin Microbiol 1985, 22:924-933.

10. Gristina AG, Oga M, Webb LX, Hobgood CD: Adherent bacterial colonization in the pathogenesis of osteomyelitis. Science 1985, 228:990-993.

11. Sedghizadeh PP, Kumar SK, Gorur A, Schaudinn C, Shuler CF, Costerton JW: Microbial biofilms in osteomyelitis of the jaw and osteonecrosis of the jaw secondary to bisphosphonate therapy. J Am Dent Assoc 2009, 140:1259-1265. 
12. Esteban J, Molina-Manso D, Spiliopoulou I, Cordero-Ampuero J, FernandezRoblas R, Foka A, Gomez-Barrena E: Biofilm development by clinical isolates of Staphylococcus spp. from retrieved orthopedic prostheses. Acta Orthop 2010, 81:674-679.

13. O'Neill E, Pozzi C, Houston P, Smyth D, Humphreys H, Robinson DA, O'Gara JP: Association between methicillin susceptibility and biofilm regulation in Staphylococcus aureus isolates from device-related infections. J Clin Microbiol 2007, 45:1379-1388.

14. Sanchez CJ Jr, Mende K, Beckius ML, Akers KS, Romano DR, Wenke JC, Murray CK: Biofilm formation by clinical isolates and the implications in chronic infections. BMC Infect Dis 2013, 13:47.

15. Palmer M, Costerton W, Sewecke J, Altman D: Molecular techniques to detect biofilm bacteria in long bone nonunion: a case report. Clin Orthop Relat Res 2011, 469:3037-3042.

16. Altman DT, AltmanT GT, Sewecke JJ, Gause TM, Costerton WJ: Can We Trust Intraoperative Culture Results in Nonunions? San Antonio, TX: Orthopaedic Trauma Association; 2011. 12-15 October 2011.

17. Yasuda H, Shima N, Nakagawa N, Yamaguchi K, Kinosaki M, Mochizuki S, Tomoyasu A, Yano K, Goto M, Murakami A, et al: Osteoclast differentiation factor is a ligand for osteoprotegerin/osteoclastogenesis-inhibitory factor and is identical to TRANCE/RANKL. Proc Natl Acad Sci U S A 1998 95:3597-3602

18. Suda T, Takahashi N, Udagawa N, Jimi E, Gillespie MT, Martin TJ: Modulation of osteoclast differentiation and function by the new members of the tumor necrosis factor receptor and ligand families. Endocr Rev 1999, 20:345-357.

19. Kong YY, Yoshida H, Sarosi I, Tan HL, Timms E, Capparelli C, Morony S, Oliveira-dos-Santos AJ, Van G, Itie A, et al: OPGL is a key regulator of osteoclastogenesis, lymphocyte development and lymph-node organogenesis. Nature 1999, 397:315-323.

20. Marriott I: Osteoblast responses to bacterial pathogens: a previously unappreciated role for bone-forming cells in host defense and disease progression. Immunol Res 2004, 30:291-308.

21. Montanaro L, Testoni F, Poggi A, Visai L, Speziale P, Arciola CR: Emerging pathogenetic mechanisms of the implant-related osteomyelitis by Staphylococcus aureus. Int J Artif Organs 2011, 34:781-788.

22. Boyce BF, Xing L: Functions of RANKL/RANK/OPG in bone modeling and remodeling. Arch Biochem Biophys 2008, 473:139-146.

23. Claro T, Widaa A, O'Seaghdha M, Miajlovic H, Foster TJ, O'Brien FJ, Kerrigan SW: Staphylococcus aureus protein A binds to osteoblasts and triggers signals that weaken bone in osteomyelitis. PLoS One 2011, 6:e18748.

24. Widaa A, Claro T, Foster TJ, O'Brien FJ, Kerrigan SW: Staphylococcus aureus Protein A Plays a Critical Role in Mediating Bone Destruction and Bone Loss in Osteomyelitis. PLoS One 2012, 7:e40586.

25. Smeltzer MS, Thomas JR, Hickmon SG, Skinner RA, Nelson CL, Griffith D, Parr TR Jr, Evans RP: Characterization of a rabbit model of staphylococcal osteomyelitis. J Orthop Res 1997, 15:414-421.

26. Weiss EC, Zielinska A, Beenken KE, Spencer HJ, Daily SJ, Smeltzer MS: Impact of sarA on daptomycin susceptibility of Staphylococcus aureus biofilms in vivo. Antimicrob Agents Chemother 2009, 53:4096-4102.

27. Secor PR, James GA, Fleckman P, Olerud JE, McInnerney K, Stewart PS: Staphylococcus aureus Biofilm and Planktonic cultures differentially impact gene expression, mapk phosphorylation, and cytokine production in human keratinocytes. BMC Microbiol 2011, 11:143.

28. Christensen GD, Simpson WA, Younger JJ, Baddour LM, Barrett FF, Melton $\mathrm{DM}$, Beachey $\mathrm{EH}$ : Adherence of coagulase-negative staphylococci to plastic tissue culture plates: a quantitative model for the adherence of staphylococci to medical devices. J Clin Microbiol 1985, 22:996-1006.

29. Shevchenko A, Wilm M, Vorm O, Jensen ON, Podtelejnikov AV, Neubauer G, Mortensen P, Mann M: A strategy for identifying gel-separated proteins in sequence databases by MS alone. Biochem Soc Trans 1996, 24:893-896.

30. van Engeland M, Ramaekers FC, Schutte B, Reutelingsperger CP: A novel assay to measure loss of plasma membrane asymmetry during apoptosis of adherent cells in culture. Cytometry 1996, 24:131-139.

31. Xiao Y, Zhong Y, Greene W, Dong F, Zhong G: Chlamydia trachomatis infection inhibits both Bax and Bak activation induced by staurosporine. Infect Immun 2004, 72:5470-5474.

32. Stephens AS, Stephens SR, Morrison NA: Internal control genes for quantitative RT-PCR expression analysis in mouse osteoblasts, osteoclasts and macrophages. BMC Res Notes 2011, 4:410.

33. Arciola CR, Hansch GM, Visai L, Testoni F, Maurer S, Campoccia D, Selan L, Montanaro L: Interactions of staphylococci with osteoblasts and phagocytes in the pathogenesis of implant-associated osteomyelitis. Int J Artif Organs 2012, 10:713-726.

34. Claro T, Widaa A, McDonnell C, Foster TJ, O'Brien FJ, Kerrigan SW: Staphylococcus aureus protein A binding to osteoblast tumour necrosis factor receptor 1 results in activation of nuclear factor kappa B and release of interleukin-6 in bone infection. Microbiology 2013, 159:147-154.

35. Ning RD, Zhang XL, Li QT, Guo XK: The effect of Staphylococcus aureus on apoptosis of cultured human osteoblasts. Orthop Surg 2011 3:199-204.

36. Percival SL, Hill KE, Malic S, Thomas DW, Williams DW: Antimicrobial tolerance and the significance of persister cells in recalcitrant chronic wound biofilms. Wound Repair Regen 2011, 19:1-9.

37. Thomson CH: Biofilms: do they affect wound healing? Int Wound J 2011, 8:63-67.

38. Wolcott $R$, Dowd $S$ : The role of biofilms: are we hitting the right target? Plast Reconstr Surg 2011, 127(Suppl 1):28S-35S.

39. Kirker KR, James GA, Fleckman P, Olerud JE, Stewart PS: Differential effects of planktonic and biofilm MRSA on human fibroblasts. Wound Repair Regen 2012, 20:253-261.

40. James GA, Swogger E, Wolcott R, Pulcini E, Secor P, Sestrich J, Costerton JW, Stewart PS: Biofilms in chronic wounds. Wound Repair Regen 2008, 16:37-44.

41. Stephens P, Wall IB, Wilson MJ, Hill KE, Davies CE, Hill CM, Harding KG, Thomas DW: Anaerobic cocci populating the deep tissues of chronic wounds impair cellular wound healing responses in vitro. $\mathrm{Br} J$ Dermatol 2003, 148:456-466.

42. Young AB, Cooley ID, Chauhan VS, Marriott I: Causative agents of osteomyelitis induce death domain-containing TNF-related apoptosisinducing ligand receptor expression on osteoblasts. Bone 2011, 48:857-863.

43. McCall SH, Sahraei M, Young AB, Worley CS, Duncan JA, Ting JP, Marriott I: Osteoblasts express NLRP3, a nucleotide-binding domain and leucinerich repeat region containing receptor implicated in bacterially induced cell death. J Bone Miner Res 2008, 23:30-40.

44. Thomas VC, Hancock LE: Suicide and fratricide in bacterial biofilms. Int J Artif Organs 2009, 32:537-544.

45. Bayles KW: The biological role of death and lysis in biofilm development. Nat Rev Microbiol 2007, 5:721-726.

46. DeLeo FR, Diep BA, Otto M: Host defense and pathogenesis in Staphylococcus aureus infections. Infect Dis Clin North Am 2009, 23:17-34

47. Walev I, Martin E, Jonas D, Mohamadzadeh M, Muller-Klieser W, Kunz L, Bhakdi S: Staphylococcal alpha-toxin kills human keratinocytes by permeabilizing the plasma membrane for monovalent ions. Infect Immun 1993, 61:4972-4979.

48. Walev I, Palmer M, Martin E, Jonas D, Weller U, Hohn-Bentz H, Husmann M, Bhakdi S: Recovery of human fibroblasts from attack by the pore-forming alpha-toxin of Staphylococcus aureus. Microb Pathog 1994, 17:187-201.

49. Craven RR, Gao X, Allen IC, Gris D, Bubeck Wardenburg J, McElvania-Tekippe E, Ting JP, Duncan JA: Staphylococcus aureus alpha-hemolysin activates the NLRP3-inflammasome in human and mouse monocytic cells. PLoS One 2009, 4:e7446.

50. Smagur J, Guzik K, Magiera L, Bzowska M, Gruca M, Thogersen IB, Enghild JJ, Potempa J: A new pathway of staphylococcal pathogenesis: apoptosislike death induced by Staphopain B in human neutrophils and monocytes. J Innate Immun 2009, 1:98-108.

51. Smagur J, Guzik K, Bzowska M, Kuzak M, Zarebski M, Kantyka T, Walski M, Gajkowska B, Potempa J: Staphylococcal cysteine protease staphopain B ( $\mathrm{SspB}$ ) induces rapid engulfment of human neutrophils and monocytes by macrophages. Biol Chem 2009, 390:361-371.

52. Komori T: Regulation of bone development and extracellular matrix protein genes by RUNX2. Cell Tissue Res 2010, 339:189-195.

53. Komori T: Regulation of osteoblast differentiation by Runx2. Adv Exp Med Biol 2010, 658:43-49.

54. Hofbauer LC, Kuhne CA, Viereck V: The OPG/RANKL/RANK system in metabolic bone diseases. J Musculoskelet Neuronal Interact 2004, 4:268-275.

\section{doi:10.1186/1471-2474-14-187}

Cite this article as: Sanchez et al:: Staphylococcus aureus biofilms decrease osteoblast viability, inhibits osteogenic differentiation, and increases bone resorption in vitro. BMC Musculoskeletal Disorders 2013 14:187. 